\title{
Pseudogene KRT17P3 drives cisplatin resistance of human NSCLC cells by modulating miR-497-5p/mTOR
}

\author{
Zhibo Hou ${ }^{1,2,3}$ | Yi Wang ${ }^{4,5}$ | Ning Xia ${ }^{3}$ | Tangfeng Lv ${ }^{1,2}$ | Xiaoqin Yuan ${ }^{4,5}$ (D) | \\ Yong Song ${ }^{1,2}$ (D)
}

${ }^{1}$ Department of Respiratory Medicine, Jinling Hospital, Nanjing University School of Medicine, Nanjing, China

${ }^{2}$ Nanjing University Institute of Respiratory Medicine, Nanjing, China

${ }^{3}$ Department of Respiratory Medicine, Nanjing Brain Hospital, Nanjing Medical University, Nanjing, China

${ }^{4}$ Department of Anatomy, Histology and Embryology, Nanjing Medical University, Nanjing, China

${ }^{5}$ Key Laboratory for Aging and Disease, Nanjing Medical University, Nanjing, China

\section{Correspondence}

Xiaoqin Yuan, Department of Anatomy, Histology and Embryology, Nanjing Medical University, \#101, Longmian Avenue, Nanjing 211166, China.

Email: yuanxq@njmu.edu.cn

Yong Song, Department of Respiratory Medicine, Jinling Hospital, Nanjing University School of Medicine, \#305, East Zhongshan Road, Nanjing 210002, China.

Email:yong.song@nju.edu.cn

\section{Funding information}

Nanjing Science and Technology Commission, Grant/Award Number: 201715059; Jiangsu Provincial Medical Youth Talent, Grant/Award Number: QNRC2016126; National Natural Science Foundation of China, Grant/Award Number: 81572937,81770082 and 81772500

\begin{abstract}
Chemoresistance is a major obstacle in non-small cell lung cancer (NSCLC) treatment. The pseudogene keratin 17 pseudogene 3 (KRT17P3) has been previously shown to be upregulated in lung cancer tissues of patients with cisplatin resistance. In the present study, RT-qPCR was performed to evaluate KRT17P3 levels in plasma samples collected from 30 cisplatin-resistant and 32 cisplatin-sensitive patients. We found that the plasma level of KRT17P3 is upregulated in cisplatin-resistant patients, and the increased expression of plasma KRT17P3 is associated with poor chemotherapy response. Functional studies demonstrated that KRT17P3 overexpression in cultured NSCLC cells increases cell viability and decreases apoptosis upon cisplatin treatment in vitro and in vivo, while KRT17P3 knockdown has the opposite effect. Mechanistically, bioinformatics analysis, RNA immunoprecipitation, and dual luciferase reporter assay indicated that KRT17P3 acts as a molecular sponge for miR497-5p and relieves the binding of miR-497-5p to its target gene mTOR. Rescue experiments validated the functional interaction between KRT17P3, miR-497-5p, and mTOR. Taken together, our findings indicate that KRT17P3/miR-497-5p/mTOR regulates the chemosensitivity of NSCLC, suggesting a potential therapeutic target for cisplatin-resistant NSCLC patients. KRT17P3 may be a potential peripheral blood marker of NSCLC patients resistant to cisplatin.
\end{abstract}

\section{KEYWORDS}

Cisplatin, KRT17P3, miR-497-5p, mTOR, non-small-cell lung cancer

\section{INTRODUCTION}

Lung cancer is the leading cause of cancer-related deaths worldwide in both males and females, which can be attributed to its high incidence and mortality. ${ }^{1}$ Non-small cell lung cancer (NSCLC) accounts for $85 \%$ of lung cancer cases. Platinum-based combination chemotherapy is currently recommended as a standard treatment for patients with advanced NSCLC. ${ }^{2}$ Treatment with cisplatin plus

Abbreviations: KRT17P3, keratin 17 pseudogene 3; LUAD, lung adenocarcinoma; LUSC, lung squamous cell carcinoma; NSCLC, non-small cell lung cancer; PD, progressive disease; PR, partial response; RT-qPCR, real-time polymerase chain reaction; TCGA, The Cancer Genome Atlas.

Zhibo Hou and Yi Wang are equal contributors.

This is an open access article under the terms of the Creative Commons Attribution-NonCommercial-NoDerivs License, which permits use and distribution in any medium, provided the original work is properly cited, the use is non-commercial and no modifications or adaptations are made.

(c) 2020 The Authors. Cancer Science published by John Wiley \& Sons Australia, Ltd on behalf of Japanese Cancer Association. 
one of the new agents remains the most widely employed first-line chemotherapeutic regimen for the treatment of NSCLC. However, the individual response to drug therapy varies, and the emergence of drug resistance greatly limits long-term curative effects. Therefore, it is important to identify molecular mechanisms involved in the cisplatin chemoresistance of lung cancer.

There are almost 15000 human pseudogenes, according to GENCODE Release Annotations (version 33; http://www.genco degenes.org/stats/current.html). Pseudogenes were initially regarded as nonfunctional genomic junk resulting from inactivating gene mutations during evolution. However, recent studies have implicated dysregulation of pseudogenes in diverse physiological and pathological processes, including cancer. ${ }^{3,4}$ Pseudogenes can interact with parental genes or other gene loci, leading to alterations in sequences and/or transcriptional activities. Pseudogene-derived RNAs play multifaceted roles in posttranscriptional regulation as antisense RNAs, endogenous small-interference RNAs, and competing endogenous RNAs that govern gene expression related to cancer progression, metastasis, and drug resistance in multiple tumors. ${ }^{5-9}$ For example, recent research has revealed that the pseudogene KRT19P3 suppresses gastric cancer growth and metastasis through the COPS7A-mediated NF-кB pathway. ${ }^{7}$ NANOGP8 expression is correlated with cell proliferation, invasion, metastasis, and oxaliplatin chemoresistance in gastric cancer. ${ }^{6}$ Additionally, the pseudogene-derived long noncoding RNA SFTA1P promotes apoptosis and increases cisplatin sensitivity by regulating the hnRNP-UGADD45A axis in lung squamous cell carcinoma (LUSC). ${ }^{9}$

Our previous work demonstrated that the pseudogene KRT17P3 (keratin 17 pseudogene 3, ENSG00000231870; previously named IncRNA AC006050.3-003) is significantly downregulated in partial-response (PR) compared with progressive-disease (PD) tumor tissue samples in LUSC patients. This result was obtained by using IncRNA expression microarray data and was confirmed in another 60 LUSC patients by quantitative real-time polymerase chain reaction (RT-qPCR). ${ }^{10}$ Moreover, receiver operating characteristic curve analysis revealed that KRT17P3 constitutes a valuable biomarker for differentiating PR patients from PD patients indicating that KRT17P3 may be associated with drug resistance. KRT17P3 is located in chromosome 17:30 567 700-30 571748 reverse strand in GRCh38 coordinates. To date, no other studies have detailed the expression pattern, biological functions, or regulatory mechanisms of KRT17P3 in human cancer. Furthermore, the biological function and underlying molecular mechanism of KRT17P3 in cisplatin resistance has not been characterized. TCGA data analysis showed that the expression of KRT17P3 was increased both in lung adenocarcinoma (LUAD) and LUSC tissues, suggesting that the dysregulation of KRT17P3 is common in NSCLC. We speculated that KRT17P3 is associated with cisplatin resistance not only in LUSC but also in LUAD.

To further explore whether KRT17P3 expression is associated with resistance to chemotherapeutic agents, we collected plasma from LUAD or LUSC patients with cisplatin resistance or sensitivity, and detected KRT17P3 expression by RT-qPCR. We demonstrate that dysregulation of KRT17P3 confers cisplatin sensitivity in A549 and SK-MES-1 NSCLC cells in vitro and in a mouse model in vivo. KRT17P3 modulates cisplatin sensitivity via direct binding of miR497-5p, which regulates the target gene mTOR. Moreover, KRT17P3 expression is upregulated in peripheral blood samples of PD NSCLC patients. Taken together, these findings provide new insights into the role of KRT17P3 in cisplatin resistance.

\section{2 | MATERIALS AND METHODS}

\section{1 | Clinical samples}

Plasma was collected from 62 NSCLC patients at Jinling Hospital, Nanjing University School of Medicine from January 2016 to January 2019. The clinical information of the NSCLC patients is summarized in Table 1. The entry standards and standards for objective tumor response for target lesions used in this study were identical with those in our previous study. ${ }^{10}$ Front-line chemotherapy for LUAD comprised cisplatin $75 \mathrm{mg} / \mathrm{m}^{2}$ on day 1 , plus gemcitabine $1000 \mathrm{mg} / \mathrm{m}^{2}$ on days 1 and 8 , or plus Docetaxel $75 \mathrm{mg} /$ $\mathrm{m}^{2}$ on day 1 , every 21 days for 4 cycles. Front-line chemotherapy for LUSC comprised cisplatin $75 \mathrm{mg} / \mathrm{m}^{2}$ on day 1 , plus pemetrexed $500 \mathrm{mg} / \mathrm{m}^{2}$ on day 1 , or plus Docetaxel $75 \mathrm{mg} / \mathrm{m}^{2}$ on day 1 , every 21 days for 4 cycles. Up to $5 \mathrm{~mL}$ of blood was collected from patients in a $\mathrm{K}_{2}$ EDTA plasma tube (BD Vacutainer, Becton, Dickinson and Company) and immediately centrifuged (2000 g for $10 \mathrm{~min}$ utes) within 30 minutes after collection. After separation, plasma

TABLE 1 Clinicopathological characteristics of participants in this study

\begin{tabular}{|c|c|c|c|}
\hline & $\begin{array}{l}\text { Resistant } \\
(\mathrm{n}=30)\end{array}$ & $\begin{array}{l}\text { Sensitive } \\
(n=32)\end{array}$ & $P$ value \\
\hline \multicolumn{4}{|l|}{ Gender } \\
\hline Female & 5 & 9 & .367 \\
\hline Male & 25 & 23 & \\
\hline \multicolumn{4}{|l|}{ Age(year) } \\
\hline$<65$ & 13 & 17 & .459 \\
\hline$\geq 65$ & 17 & 15 & \\
\hline \multicolumn{4}{|l|}{ Smoking } \\
\hline No & 13 & 13 & 1.000 \\
\hline Yes & 17 & 19 & \\
\hline \multicolumn{4}{|l|}{ Histological type } \\
\hline Adenocarcinoma & 21 & 21 & .789 \\
\hline Squamous cell carcinoma & 9 & 11 & \\
\hline \multicolumn{4}{|l|}{ Tumor size (cm) } \\
\hline$<4$ & 14 & 23 & .069 \\
\hline$\geq 4$ & 16 & 9 & \\
\hline \multicolumn{4}{|l|}{ TNM stage } \\
\hline$I+I I$ & 0 & 2 & .492 \\
\hline III + IV & 30 & 30 & \\
\hline
\end{tabular}


samples were transferred to RNase- and DNase-free tubes and stored at $-80^{\circ} \mathrm{C}$ until total RNA was extracted. All samples were collected after obtaining written informed consent from the patients, in accordance with the Declaration of Helsinki. The study was approved by the Ethics Committee of Jinling Hospital, Nanjing University School of Medicine.

\section{2 | Analysis of TCGA database}

The transcriptome expression profiles of 514 LUAD tissues and 58 adjacent normal tissues as well as 494 LUSC tissues and 49 adjacent normal tissues were obtained from TCGA database (https://cance rgenome.nih.gov). These data were used to analyze differences in KRT17P3 expression between tumor and normal tissues.

\section{3 | Cell culture and reagents}

The A549 and SK-MES-1 cell lines were purchased from the Shanghai Institute of Cell Biology, Chinese Academy of Sciences and were maintained in RPMI 1640 (Gibco; for A549) or EMEM (ATCC 30-2003; for SK-MES-1) supplemented with $10 \%$ FBS (Gibco), $100 \mathrm{U} / \mathrm{mL}$ penicillin, and $0.1 \mathrm{mg} / \mathrm{mL}$ streptomycin (Gibco). All cell lines were cultured in $5 \%$ $\mathrm{CO}_{2}$ at $37^{\circ} \mathrm{C}$. The cells were treated with cisplatin (Sigma-Aldrich) for different periods of time. Hsa-miR-497-5p mimic and miR-497-5p inhibitor, and control mimic and inhibitor were purchased from RiboBio.

\section{4 | RNA extraction and RT-qPCR}

Total RNA was extracted from cultured cells with TRIzol reagent (Invitrogen) according to the manufacturer's protocol. Plasma RNA was extracted using miRNeasy Serum/Plasma Kit (Qiagen, Inc) according to the manufacturer's protocol. cDNA was synthesized with the PrimeScript 1st Strand cDNA Synthesis Kit (Takara Bio) according to the user's manual. RT-qPCR analysis with gene-specific primers was performed to determine the relative expression of KRT17P3 using SYBR green reagents (Takara Bio) in an ABI 7300 sequence detector (Applied Biosystems). GAPDH mRNA was used for normalization. The PCR primers used in this study were as follows: KRT17P3, forward: 5'- CAGCAGAACCAGCAGTACAAG-3' and reverse: 5'- TCAGCCACGAAGATGCTTAT-3'; keratin 17, forward: 5'-CCAGGATGGCAAGGTCATCTC-3' and reverse: 5'- GTCATCAGGC AAGGAAGCAT-3'; and ACTIN, forward: 5'-TCATGAAGTGTGACGTG GACAT-3' and reverse: 5'- CTCAGGAGGAGCAATGATCTTG-3'.

\section{5 | Cell Counting Kit-8 (CCK-8) assay}

Cells transfected with plasmid or infected with lentivirus were seeded into 96 -well plates at a density of $5 \times 10^{3}$ cells. After cellular adhesion, medium containing cisplatin at distinct concentrations
(0-14 $\mu \mathrm{g} / \mathrm{mL})$ was added to the corresponding cells. After $72 \mathrm{~h}$, cell viability was determined by CCK-8 (Dojindo) according to the manufacturer's protocol. Absorbance at $450 \mathrm{~nm}$ was measured using a microplate reader (Bio-Tek). The IC50 was calculated with GraphPad software.

\section{6 | Cell apoptosis analysis}

Cells were treated with different concentrations of cisplatin for 24 hours, stained with FITC Annexin $\mathrm{V}$ and propidium iodide (BD Biosciences) in accordance with the manufacturer's instructions, and then analyzed by FACScan using CellQuest software (BD Biosciences).

\section{7 | Generation of KRT17P3 knockdown or overexpression cells}

Lentivirus harboring shRNA-KRT17P3 and KRT17P3 expression plasmids were constructed by Genechem. After 72 hours of infection, the cells were subjected to further studies. Cells infected with lentivirus expressing scrambled shRNA or transfected with a puromycin resistance plasmid were used as control. The targeting sequence of the KRT17P3 shRNA was 5'-TGAACGAGATGCATGAGTA-3'. For the tumorigenicity studies, stable A549 cells with KRT17P3 overexpression were selected in puromycin.

\subsection{Xenograft animal model}

BALB/c nude mice (4-5 weeks old) were maintained on a 12-hour light/12-hour dark cycle under pathogen-free conditions. After anesthetization, stable KRT17P3-overexpressing A549 cells and control cells $\left(4 \times 10^{6}\right)$ were injected subcutaneously in the back flanks of mice. On day 7 after tumor cell implantation, cisplatin was intraperitoneally injected (i.p.) twice a week. Tumor growth was examined every 7 days for 4 weeks, and the tumor volume was assessed according to the equation: volume $=$ length $\times$ width $^{2} \times 0.5$. All mice were sacrificed, and the xenografts were dissected and weighed for further studies. Animal welfare and experimental procedures were approved by the Institutional Animal Care and Use Committee of Nanjing Medical University.

\subsection{Western blot analysis}

Cells were lysed with RIPA, and $30 \mu$ g protein was separated by SDSPAGE and transferred to polyvinylidene fluoride membranes. The membranes were blotted with $5 \%$ nonfat milk and probed with primary antibodies against anti-PARP-1 (Abcam), anti-mTOR (Abcam), anti-phosphory-mTOR (Abcam), or anti-GAPDH, followed by the appropriate secondary antibodies and chemiluminescent detection. 


\subsection{0 | Luciferase reporter assay}

The sequences of KRT17P3 containing the predicted binding sites (5'-GACAAUGCCCAUCUGGCUGCUGCUGA-3') for miR-497-5p or the respective mutated sequence (5'-GACAAUGCCCAUCUGGCGUA GUAGGA-3') were amplified by PCR (forward primer: 5'-GATCG CCGTGTAATTCTAGAGCCAGTACTACAGGACAATCGAG-3', reverse primer:5'-CCGGCCGCCCCGACTCTAGATGGCCAAGGTCAGCCCAT CCAGC-3'). The 247-bp fragment of either KRT17P3-WT or KRT17P3-MUT was subcloned into the GV272 vector (Genechem) for luciferase reporter assay using a one-step directed cloning kit (Novoprotein) to construct plasmids KRT17P3-WT and KRT17P3MUT, respectively. These plasmids were then cotransfected into HEK293T cells with miR-497a mimic or mimic- nagative control (NC). Dual-luciferase assays (Promega) were performed according to the manufacturer's instructions. The 3'-UTR of mTOR mRNA containing the intact miRNA-497-5p family recognition sequences (AACTGAAACCATGGTGAGAAAGTTTGACTTTGTTAAATATTTT GAAATGTAAATGAAAAGAACTACTGTATATTAAAAGTTGGTTT GAACCAACTTTCTAGCTGCTGTTGAAGAATATATTGTCAGAAA CACAAGGCTTGATTTGGTTCCCAGGACAGTGAAACATAGTAA TACCACGTAAATCAAGCCATTCATTTTGGGGAACAG) or mutant sequences(AACTGAAACCATGGTGAGAAAGTTTGACTTTGTTAAA TATTTTGAAATGTAAATGAAAAGAACTACTGTATATTAAAAGT TGGTTTGAACCAACTTTCTATAGTAGTTTGAAGAATATATTGTC AGAAACACAAGGCTTGATTTGGTTCCCAGGACAGTGAAACATAG TAATACCACGTAAATCAAGCCATTCATTTTGGGGAACAG) were PCR-amplifiedand subclonedintotheSaclandSall sites of the pmirGLO vector named mTOR3'UTR-WT or mTOR3'UTR-WT, respectively.

\subsection{1 | RNA immunoprecipitation (RIP)}

RIP was performed using an EZ-Magna RIP Kit (Millipore) according to the manufacturer's instructions. Briefly, A549 cells were cotransfected with pKRT17P3 (Genechem) or control plasmid in the presence or absence of pMS2-GFP (Genechem) plasmid and were lysed with RIP lysis buffer. The lysates were incubated with magnetic beads conjugated with anti-GFP (Abcam) or nonspecific control IgG with rotation overnight at $4^{\circ} \mathrm{C}$. Then, the proteins in the immunoprecipitants were digested with protein $\mathrm{K}$ and removed. Finally, the purified RNA was detected by RT-qPCR to measure the level of miR497-5p. For anti-AGO2 RIP, cells were transfected with pmiR-497 plasmid or control plasmid and were subjected to RIP assays using an AGO2 antibody (Abcam).

\subsection{2 | Statistical analysis}

Statistical analysis was performed with SPSS 20.0 (Abbott Laboratories). All data are presented as means \pm SEM. The Student's $t$-test was used to analyze data between two groups. The chi-square test was applied to examine the clinicopathological characteristics between chemoresistant and chemosensitive patients. The Student's $t$-test was applied to examine the relationship between KRT17P3 levels and clinicopathological characteristics. A value of $P<.05$ was considered statistically significant.

\section{3 | RESULTS}

\subsection{KRT17P3 expression is increased in the plasma of chemoresistant NSCLC patients}

Our previous study has shown KRT17P3 is upregulated in cisplatinresistant lung cancer tissues. To determine whether the expression of KRT17P3 in plasma is associated with cisplatin chemoresponse, we collected plasma from 30 chemoresistant NSCLC patients and 32 chemosensitive NSCLC patients and measured the expression of KRT17P3 by RT-qPCR. As shown in Figure 1A, plasma KRT17P3 was significantly upregulated in the chemoresistant patient group, which is consistent with the results for 60 LUSC tissue samples in our previous study. ${ }^{10}$ The association between chemoresponse and KRT17P3 expression was also confirmed by the analysis of 62 samples ( $P=.031$, Table 2$)$. These results suggest that increased expression of plasma KRT17P3 is associated with poor chemotherapy response and may be useful as a chemoresistance marker for NSCLC.

We also examined the expression of KRT17P3 in NSCLC by analyzing the TCGA database. We compared data from 514 LUAD tissues and 58 normal lung tissues and found that the expression level of KRT17P3 was increased in LUAD tissues 1.61-fold $(P=.014$; Figure 1B). KRT17P3 expression was also analyzed in an independent set of 494 LUSC and 49 normal lung tissues, which confirmed an obvious increased expression in LUSC (6.16-fold; $P<.0001$; Figure 1C).

Taken together, these data indicate that KRT17P3 is highly expressed in NSCLC and is associated with poor chemotherapy response.

\section{2 | Dysregulation of KRT17P3 confers chemosensitivity to cisplatin in A549 and SK- MES- 1 cells}

We next evaluated the effect of KRT17P3 on the chemosensitivity of NSCLC cancer cells to cisplatin. We transfected specific shRNA-KRT17P3 and control shRNA-NC into A549 and SK-MES-1 cells to knock down KRT17P3 expression (Figure 2A). As shown in Figure 2A, shRNA-KRT17P3 decreased the level of KRT17P3, while it did not affect the expression level of keratin 17, which suggested that shRNA-KRT17P3 specifically targeted KRT17P3. CCK-8 assay results show that shRNA-KRT17P3 cells exhibited significantly increased cisplatin sensitivity (Figure 2B). Calculation of the IC50 for shRNA-KRT17P3 and sh-NC in A549 cells $(1.518 \pm 0.187 \mu \mathrm{M}$ versus $3.194 \pm 0.336 \mu \mathrm{M} ; P<.05)$ and SK-MES-1 cells $(6.92 \pm 0.203 \mu \mathrm{M}$ versus $9.936 \pm 0.232 ; P<.05)$ verified the effect of shRNA-KRT17P3 in increasing cisplatin sensitivity (Figure $2 \mathrm{C}$ ). 
(A)

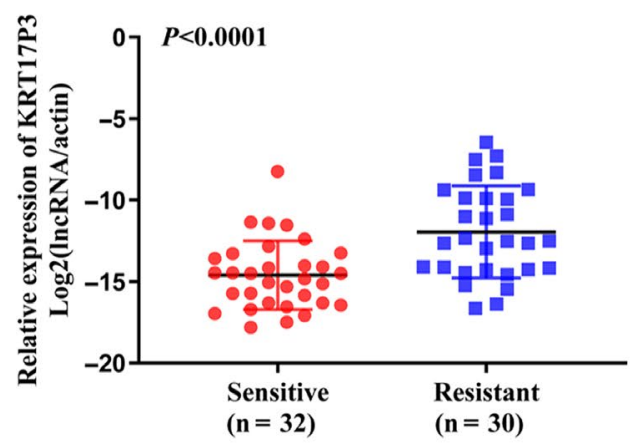

(B)

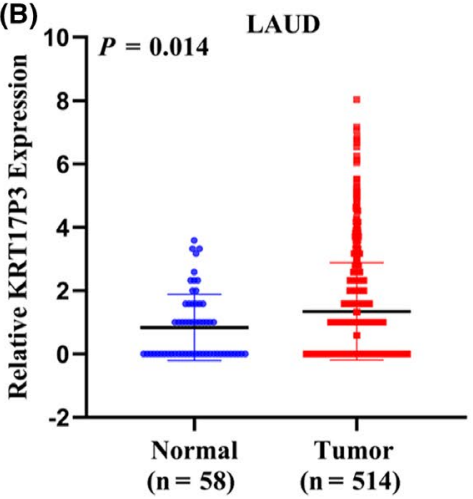

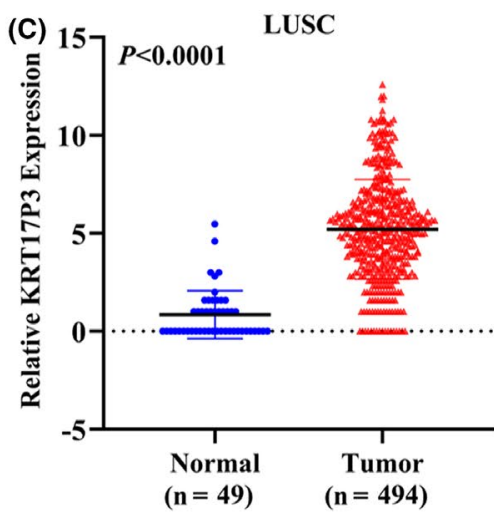

FIGURE 1 Plasma levels of KRT17P3 are upregulated in cisplatin-resistant non-small cell lung cancer (NSCLC) patients. A, The expression of keratin 17 pseudogene 3 (KRT17P3) in the plasma was increased significantly in cisplatin-resistant NSCLC patients ( $\mathrm{n}=30$ ) compared with cisplatin-sensitive patients $(n=32)$. Higher expression of KRT17P3 was detected in 514 lung adenocarcinoma (LUAD) tissues compared with 58 normal tissues (B), and in 494 lung squamous cell carcinoma (LUSC) tissues compared with 49 normal tissues (C) from The Cancer Genome Atlas (TCGA) database

TABLE 2 Association of relative expression of keratin 17 pseudogene 3 (KRT17P3) with clinicopathological parameters

\begin{tabular}{|c|c|c|c|}
\hline Feature & Cases (n) & $\begin{array}{l}\text { KRT17P3 expression } \\
\text { Log2 ( KRT17P3/ } \beta \text {-actin)(Mean } \pm \text { SD) }\end{array}$ & $P$ value \\
\hline \multicolumn{4}{|l|}{ Gender } \\
\hline Female & 14 & $-12.501 \pm 1.117$ & \multirow[t]{2}{*}{$.029^{a}$} \\
\hline Male & 48 & $-8.522 \pm 0.903$ & \\
\hline \multicolumn{4}{|l|}{ Age (yr) } \\
\hline$<65$ & 30 & $-9.912 \pm 1.026$ & \multirow[t]{2}{*}{.54} \\
\hline$\geqq 65$ & 32 & $-8.959 \pm 1.148$ & \\
\hline \multicolumn{4}{|l|}{ Smoking } \\
\hline No & 26 & $-10.114 \pm 1.123$ & \multirow[t]{2}{*}{.447} \\
\hline Yes & 36 & $-8.919 \pm 1.052$ & \\
\hline \multicolumn{4}{|l|}{ Histological type } \\
\hline Adenocarcinoma & 42 & $-10.186 \pm 1.523$ & \multirow[t]{2}{*}{.151} \\
\hline $\begin{array}{l}\text { Squamous cell } \\
\text { carcinoma }\end{array}$ & 20 & $-7.813 \pm 1.523$ & \\
\hline \multicolumn{4}{|c|}{ Chemotherapy sensitivity } \\
\hline Resistant & 30 & $-7.715 \pm 1.254$ & \multirow[t]{2}{*}{$.031^{\mathrm{a}}$} \\
\hline Sensitive & 32 & $-11.019 \pm 0.840$ & \\
\hline \multicolumn{4}{|l|}{ Tumor size(cm) } \\
\hline$<4$ & 37 & $-10.068 \pm 0.939$ & \multirow[t]{2}{*}{.309} \\
\hline$\geq 4$ & 25 & $-8.461 \pm 1.306$ & \\
\hline \multicolumn{4}{|l|}{ TNM stage } \\
\hline$I+I I$ & 2 & $-11.655 \pm 3.965$ & \multirow[t]{2}{*}{.600} \\
\hline$I I I+I V$ & 60 & $-9.346 \pm 0.787$ & \\
\hline
\end{tabular}

${ }^{a}$ For the analysis of the association of KRT17P3 levels and clinicopathological features, Student's t-test was used. $P<0.05$
As apoptosis is one of the important characteristics of chemosensitivity, we evaluated the effect of KRT17P3 on apoptosis. The results show that for both A549 and SK-MES-1 cells, the apoptotic rate of shRNA-KRT17P3-expressing cells with cisplatin treatment was significantly higher than that of shRNA-NC-expressing cells (both $P<.01$ ) (Figure 2D). Interestingly, even in cells without cisplatin treatment, there was a difference between sh-KRT17P3 and sh-NC cells in terms of the fraction of Annexin V-positive cells (Figure 2D). To confirm these findings, we further observed the levels of cleaved PARP-1 in shRNA-KRT17P3 and shRNA-NC cells with or without cisplatin treatment. Consistent with the results from Figure 2C, KRT17P3 shRNA enhanced cleaved PARP-1 levels in the presence of cisplatin 


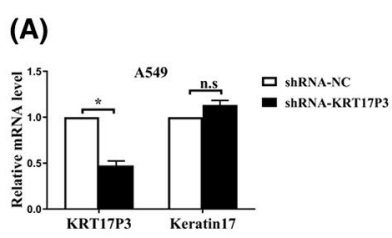

(B)

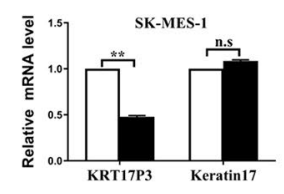

(C)

\begin{tabular}{|l|l|l|}
\hline & A549+Cisplatin & SK-MES-1+Cisplatin \\
\hline shRNA-Ctrl/ IC50 $(\mu \mathrm{g} / \mathrm{ml})$ & $3.194 \pm 0.336$ & $9.936 \pm 0.232$ \\
\hline $\begin{array}{l}\text { shRNA-KRT17P3/ IC50 } \\
(\mu \mathrm{g} / \mathrm{ml})\end{array}$ & $1.518 \pm 0.187$ & $6.92 \pm 0.203$ \\
\hline$P$ value & $<0.05$ & $<0.05$ \\
\hline
\end{tabular}
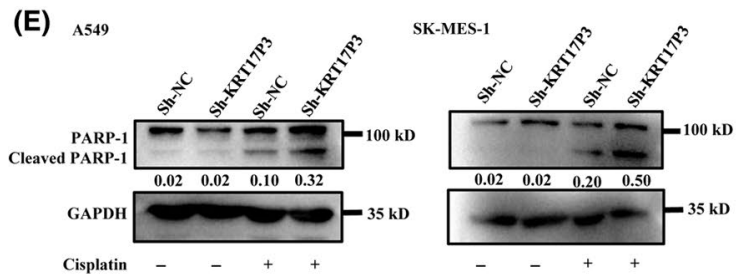
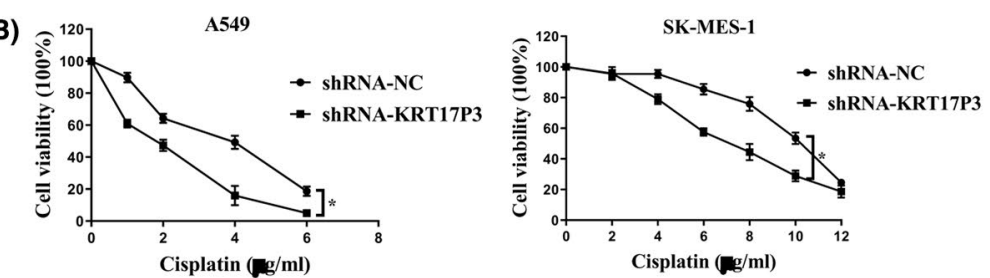

(D)
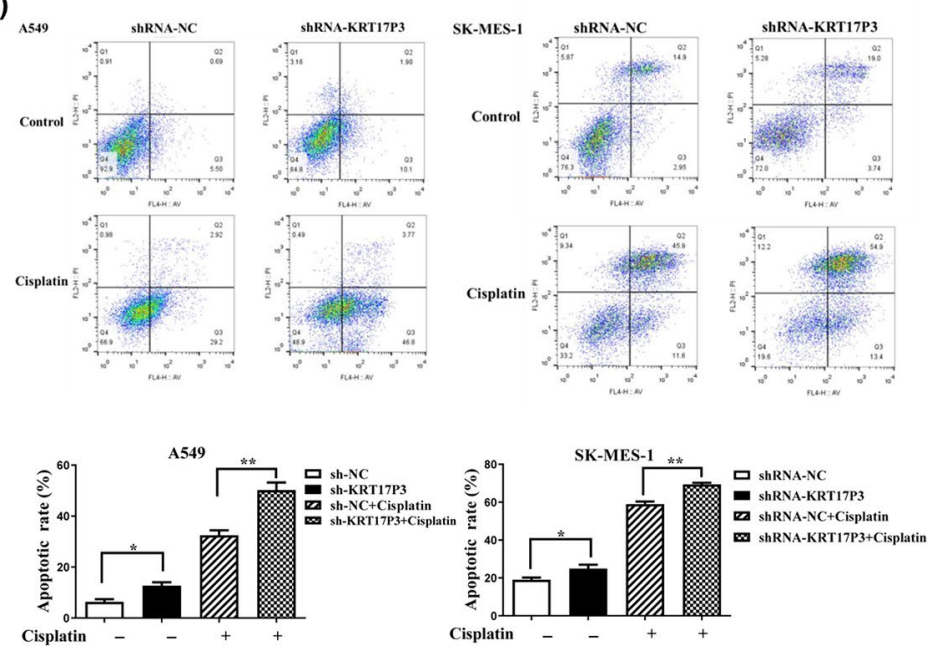

FIGURE 2 Knockdown of keratin 17 pseudogene 3 (KRT17P3) decreases cell vitality and increases apoptosis induced by cisplatin. A, KRT17P3 and keratin 17 expression were detected by RT-qPCR in A549 and SK-MES-1 cells transfected with the indicated shRNA. B, Cell counting kit-8 (CCK-8) viability assays were performed in KRT17P3 knockdown or control A549 and SK-MES-1 cells exposed to various concentrations of cisplatin for $72 \mathrm{~h}$. C, Knockdown of KRT17P3 decreased the IC50 of cisplatin on A549 and SK-MES-1 cells. D and E, Cells treated with $5 \mu \mathrm{g} / \mathrm{mL}$ (A549) or $8 \mu \mathrm{g} / \mathrm{mL}$ (SK-MES-1) cisplatin for $24 \mathrm{~h}$ were evaluated by flow cytometry for apoptotic cells (D) and measured by Western blotting for apoptosis-related PARP-1 levels (E). Error bars indicate means \pm SEM ${ }^{*} P<.05,{ }^{* *} P<.01$

exposure (Figure 2E). These results suggest that the chemosensitivity of A549 and SK-MES-1 cells is increased by KRT17P3 knockdown, which is also associated with decreased cell proliferation.

We also investigated the effect of overexpressing KRT17P3 on chemosensitivity using KRT17P3-expressing lentivirus (LvKRT17P3) (Figure 3A). CCK-8 assay results show that overexpression of KRT17P3 significantly increased the cisplatin-induced cell viability (Figure $3 \mathrm{~B}$ ). We measured the IC50 and found that KRT17P3-overexpressing cells were more resistant to cisplatin than the control cells $(6.139 \pm 0.18 \mu \mathrm{M}$ versus $3.887 \pm 0.16 \mu \mathrm{M}$ for $\mathrm{A} 549$, $P<.01 ; 14.44 \pm 0.136 \mu \mathrm{M}$ versus $11.28 \pm 0.136 \mu \mathrm{M}$ for SK-MES-1, $P<.05)$ (Figure 3C). Conversely to the KRT17P3-knockdown results, overexpression of KRT17P3 reduced the amount of cell apoptosis and cleaved PARP-1 both in cisplatin-treated and untreated cells (Figure 3D,E). These results suggest that overexpression of KRT17P3 contributes to cisplatin chemoresistance in A549 and SKMES-1 cells.

\section{3 | Dysregulation of KRT17P3 confers chemosensitivity to cisplatin in vivo}

To determine whether KRT17P3 has an effect on the in vivo resistance of NSCLC to cisplatin, we injected KRT17P3-overexpressing or control A549 cells subcutaneously into the flanks of 5-week-old nude mice. Starting at 1 week after inoculation, cisplatin was administered by intraperitoneal injection twice a week for 3 weeks to half of the mice. Compared with the cisplatin-treated control xenograft tumors, the tumors grown from Lv-KRT17P3 A549 cells in cisplatin-treated mice had larger mean volumes, faster growth, and heavier weights (Figure $4 \mathrm{~A}-\mathrm{C}$ ). Therefore, these results verify that KRT17P3 reduces the effect of cisplatin in suppressing NSCLC cell growth.

\section{4 | KRT17P3 contributes to cisplatin resistance of NSCLC cells by negatively regulating miRNA-497-5p}

A growing volume of literature has proposed that pseudogene transcripts can regulate transcription by sequestering shared microRNAs (miRNAs), thus acting as competing endogenous RNAs (ceRNAs). ${ }^{11}$ We performed a search for miRNAs that have complementary base pairing with KRT17P3 (ENSG00000231870), using the online software program miRcode (http://www.miRco de.org). Furthermore, to identify putative functions for the miRNAs in chemoresistance, we performed miRNA target prediction and evaluation using the online software program bibiserv (https:// bibiserv.cebitec.uni-bielefeld.de/rnahybrid/). The search results identified nine miRNA families that may form complementary base pairing with KRT17P3 (Figure S1 and Figure S2). Given its reported 
(A)

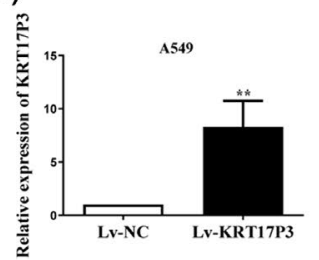

(C)

\begin{tabular}{|l|l|l|}
\hline & A549+Cisplatin & SK-MES-1+Cisplatin \\
\hline Lv-NC/ IC50 $(\mu \mathrm{g} / \mathrm{ml})$ & $3.887 \pm 0.16$ & $11.28 \pm 0.136$ \\
\hline $\begin{array}{l}\text { Lv-KRT17P3/ IC50 } \\
(\mu \mathrm{g} / \mathrm{ml})\end{array}$ & $6.139 \pm 0.18$ & $14.44 \pm 1.06$ \\
\hline$P$ value & $<0.05$ & $<0.05$ \\
\hline
\end{tabular}

(E)
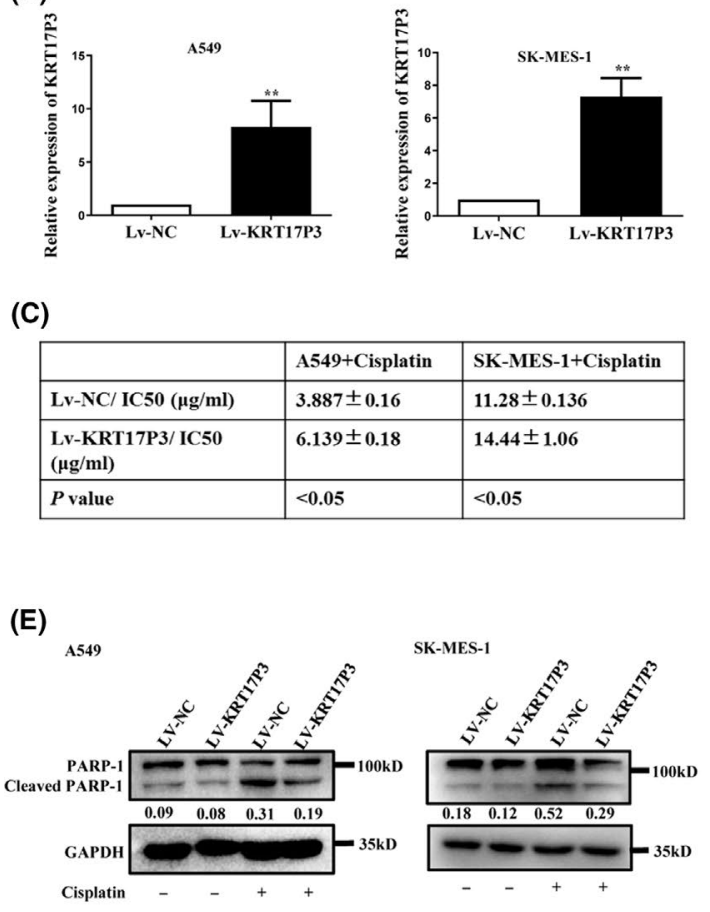

(B)
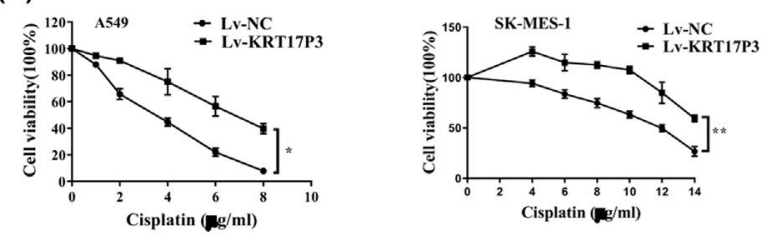

(D)
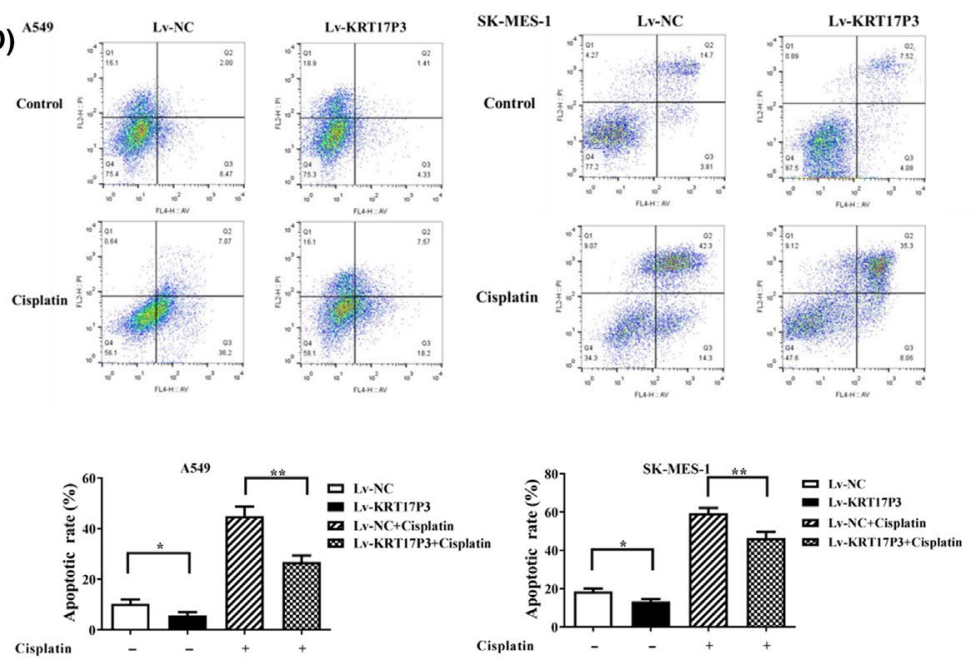

FI GURE 3 Overexpression of keratin 17 pseudogene 3 (KRT17P3) increases cell viability and decreases apoptosis induced by cisplatin. A, A549 and SK-MES-1 cells were infected with Lv-KRT17P3, and KRT17P3 expression was detected by RT-qPCR. B, KRT17P3-overexpressing A549 and SK-MES-1 cells were treated with various concentrations of cisplatin for $72 \mathrm{~h}$ and evaluated by CCK8 assay to analyze cell viability. C, Overexpression of KRT17P3 increased the IC50 of cisplatin in A549 and SK-MES-1 cells. D and E, Flow cytometry (D) and Western blotting (E) were performed to measure the apoptotic cells after $24 \mathrm{~h}$ treatment with cisplatin in KRT17P3-overexpressing A549 and SKMES-1 cells. Error bars indicate means \pm SEM. ${ }^{*} P<.05,{ }^{*} P<.01$

(A)

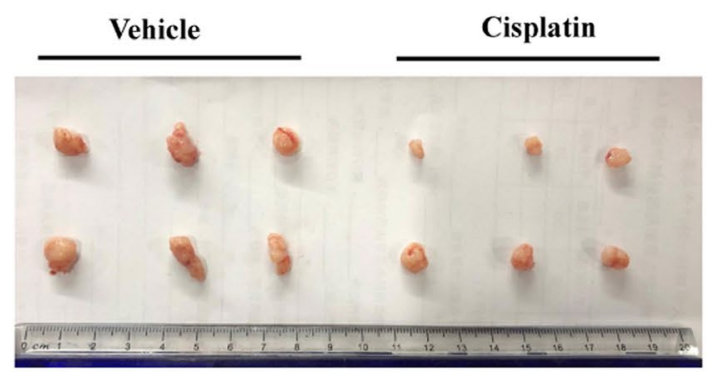

(C)

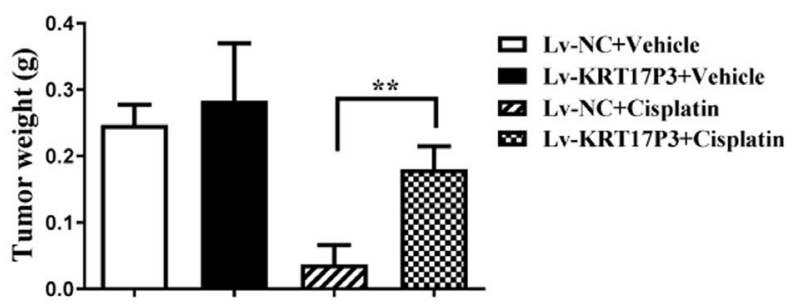

(B)

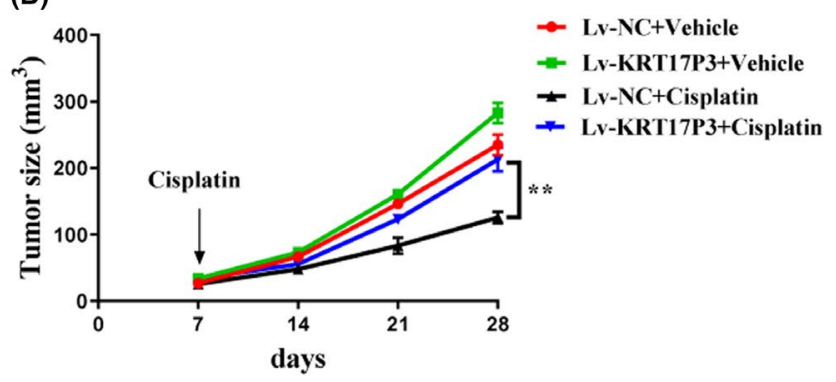

FIGURE 4 Keratin 17 pseudogene 3 (KRT17P3) enhances the cisplatin resistance of non-small cell lung cancer (NSCLC) in vivo. A, One week after subcutaneous injection with control or stable KRT17P3-overexpressing A549 cells, cisplatin or PBS were intraperitoneally injected twice a week for $3 \mathrm{wk}$. The tumors were dissected $4 \mathrm{wk}$ after subcutaneous injection and photographed. B, Tumor size was measured with calipers and calculated as the length $\times$ width $^{2} \times 0.5$ every week after 1 wk of injection. C, Weights of xenograft tumors were measured at the time of excision. Error bars indicate means \pm SEM ${ }^{*} P<.05,{ }^{* *} P<.01$ 
(A)

\begin{tabular}{|c|c|c|c|}
\hline \multirow[t]{3}{*}{ KRRT17P3 } & $5^{\prime} \mathrm{G}$ & UGC & CUG $\mathrm{C}$ \\
\hline & ACAA & CCAU & G UGCUGCUG \\
\hline & UGUU & GGUG & C ACGACGAC \\
\hline miRNA & 3' U & UCA & \\
\hline
\end{tabular}

(C)
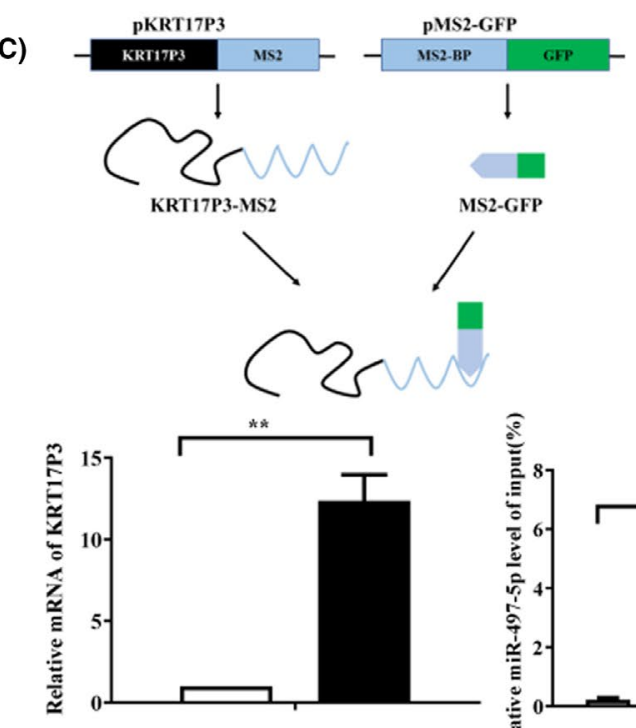

\section{pMS2-GFP} pcDNA3.1 pKRTI7P3

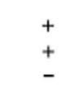

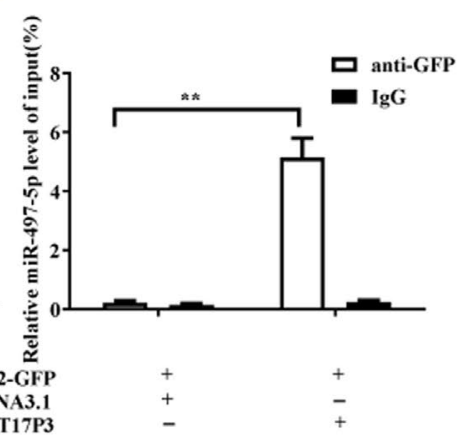

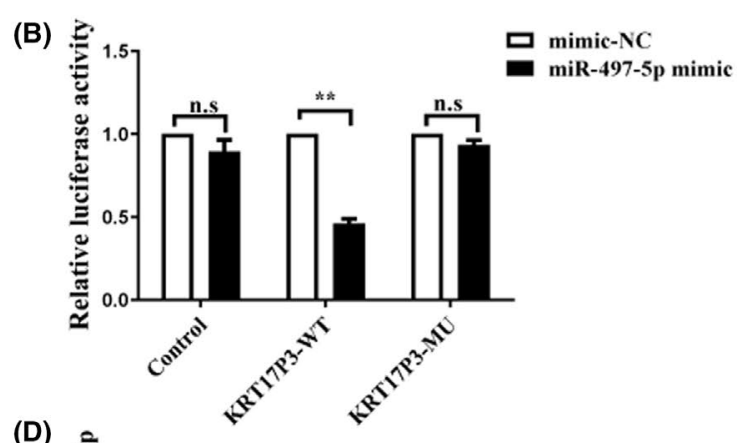

(D)
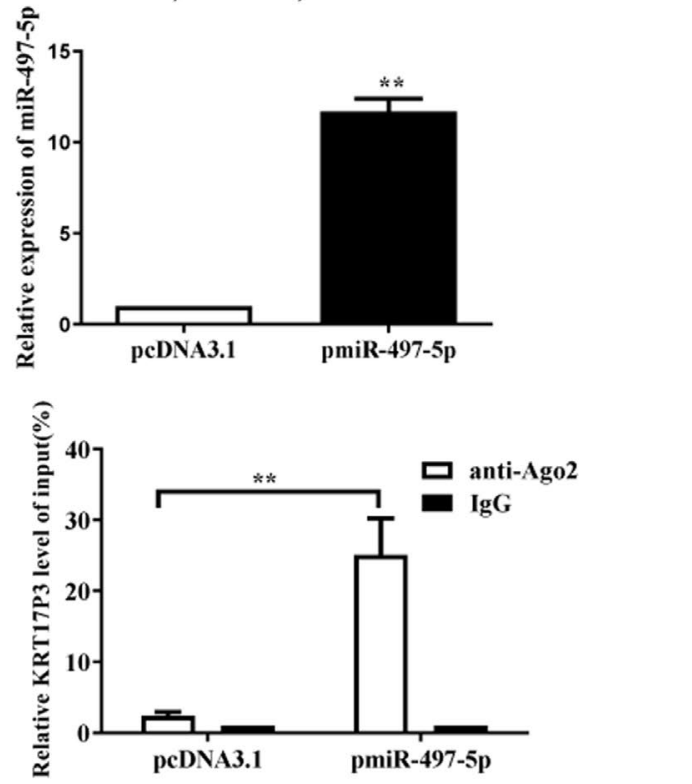

FIGURE 5 Keratin 17 pseudogene 3 (KRT17P3) reduces cisplatin sensitivity through negative regulation of miRNA-497-5p. A, Sequence of miR-497-5p predicted to target KRT17P3. B, 293T cells were cotransfected with miR-497-5p mimic or mimic-NC and plasmid with wildtype or mutant KRT17P3, and then dual-luciferase assays were performed. C, KRT17P3-MS2 and pMS2-GFP plasmids were constructed (upper panel). RNA immunoprecipitation (RIP) assay was performed using anti-GFP and IgG as a negative control in A549 cells transfected with pKRT17P3 or pcDNA3.1 in the presence of pMS2-GFP. KRT17P3 expression level was measured (low left panel). The enrichment of miR-497-5p was detected using RT-qPCR (low right panel). D, Plasmid expressing miR-497-5p was transfected into A549 cells, and RT-qPCR was done to measure the miR-497-5p expression (upper panel). RIP assay was performed using anti-Ago2, with IgG as a negative control. The enrichment of KRT17P3 was detected using RT-qPCR (low panel)

involvement in cisplatin resistance, ${ }^{12,13}$ we focused on miR-497-5p in subsequent studies. The binding site between KRT17P3 and miRNA-497-5p is shown in Figure 5A.

To confirm the interaction between miRNA-497-5p and KRT17P3, we constructed KRT17P3 luciferase reporter plasmids containing wildtype and mutant-binding sites for miR-497-5p and transfected them into $293 \mathrm{~T}$ cells together with miR-497-5p mimic or a control mimic. The miR-497-5p mimic reduced the luciferase activity of the wild-type but not the mutant plasmid (Figure 5B). To further verify the direct interaction between KRT17P3 and miR-497-5p, we overexpressed MS2-GFPtagged KRT17P3 in A549 cells and performed GFP-RIP. The precipitated miRNAs were analyzed by real-time PCR. The results suggest that the MS2-GFP-tagged KRT17P3 was specifically enriched for miR-497-5p (Figure 5C). Then we further conducted anti-Ago2 RIP in A549 cells transiently overexpressing miR-497-5p. Endogenous KRT17P3 pulldown by AGO2 antibody was specifically enriched in miR-497-5p-transfected cells but not in empty vector-transfected cells (Figure 5D). These data suggested that miR-497-5p is a miRNA binding partner of KRT17P3.
To confirm that miR-497-5p is involved in KRT17P3-mediated cisplatin resistance of NSCLC cells, we suppressed the function of miR-497-5p with miRNA inhibitors in shRNA-KRT17P3 cells. The miR-497-5p inhibitor attenuated the cisplatin sensitivity with increase in cell viability (Figure 6A) and IC50 (Figure 6B) for shKRT17P3 cells, compared with the control cells transfected with inhibitor-NC. Meanwhile, miR-497-5p inhibitor alone enhanced cell viability and IC50. Consistently, the miR-497-5p inhibitor also decreased cisplatin-induced apoptosis and cleaved PARP-1 in sh-KRT17P3 cells (Figure 6C,D). Conversely, increased miR-497-5p expression via the use of miRNA mimic reversed the cisplatin resistance in LV-KRT17P3 cells compared with cells transfected with mimic-NC, with decreased cell viability and IC50 (Figure 7A,B). The miR-497-5p mimic also increased cisplatin-induced apoptosis and expression level of cleaved PARP-1 in Lv-KRT17P3 cells (Figure 7C,D). These data suggest that miR497-5p plays an important role in KRT17P3-dependent cisplatin resistance. 
(A)

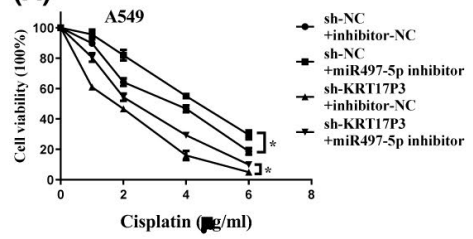

(B)

\begin{tabular}{|l|l|l|}
\hline & A549+Cisplatin & SK-MES-1+Cisplatin \\
\hline $\begin{array}{l}\text { sh-NC+inhibitor-NC/ IC50 } \\
(\mu \mathrm{g} / \mathrm{ml})\end{array}$ & $2.31 \pm 0.218$ & $10.06 \pm 0.104$ \\
\hline $\begin{array}{l}\text { sh-NC+miR497-5p inhibitor/ } \\
\text { IC50 }(\mu \mathrm{g} / \mathrm{ml})\end{array}$ & $4.104 \pm 0.14$ & $12.06 \pm 0.098$ \\
\hline$P$ value & $<0.05$ & $<0.05$ \\
\hline $\begin{array}{l}\text { sh-KRT17P3+inhibitor-NC/ } \\
\text { IC50 }(\mu \mathrm{g} / \mathrm{ml})\end{array}$ & $1.694 \pm 0.12$ & $6.167 \pm 0.183$ \\
\hline $\begin{array}{l}\text { sh-KRT17P3+miR497-5p } \\
\text { inhibitor/ IC50 }(\mu \mathrm{g} / \mathrm{ml})\end{array}$ & $2.036 \pm 0.125$ & $8.393 \pm 0.179$ \\
\hline$P$ value & $<0.05$ & $<0.05$ \\
\hline
\end{tabular}

(D) A549

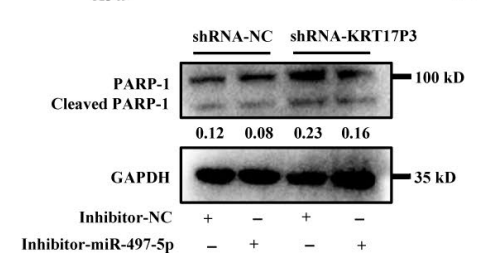

SK-MES-1

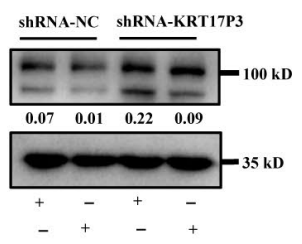

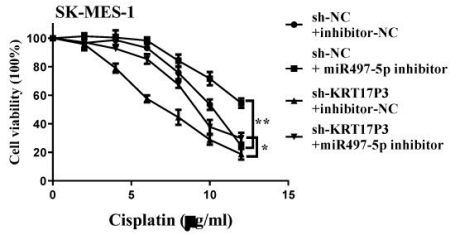

(C)

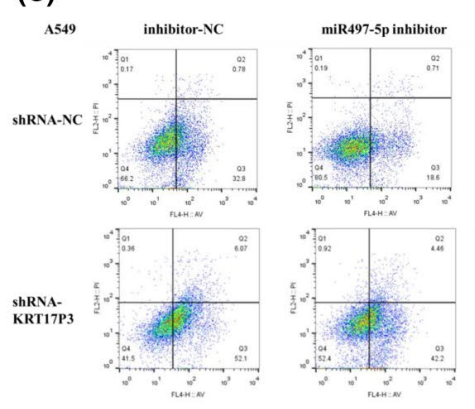

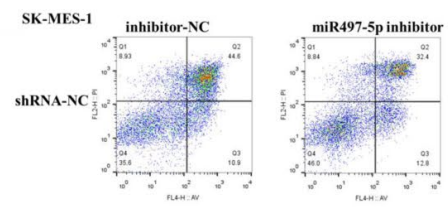

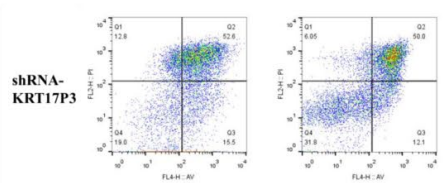

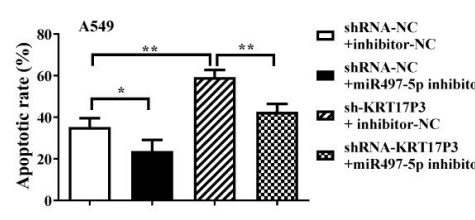

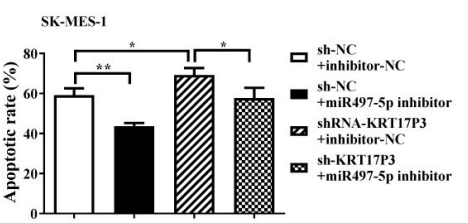

FIGURE 6 The effect of keratin 17 pseudogene 3 (KRT17P3) on cisplatin sensitivity is mediated in part through negative regulation of miRNA-497-5p. A and B, KRT17P3-knockdown A549 and SK-MES-1 cells were transfected with miR-497-5p inhibitor or control inhibitor and exposed to various concentration of cisplatin for $72 \mathrm{~h}$ to analyze cell viability and IC 50 by CCK8 assay. C and D, Apoptosis levels were evaluated by flow cytometry (C) and Western blotting (D)

(A)
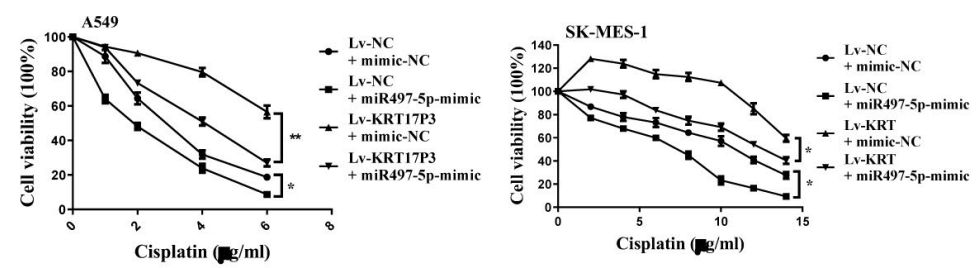

(B)

\begin{tabular}{|l|l|l|}
\hline & A549+Cisplatin & SK-MES-1+Cisplatin \\
\hline Lv-NC+mimic-NC/IC50 $(\mu \mathrm{g} / \mathrm{ml})$ & $2.279 \pm 0.16$ & $8.357 \pm 0.27$ \\
\hline $\begin{array}{l}\text { Lv-NC+miR497-5p mimic/ IC50 } \\
(\mu \mathrm{g} / \mathrm{ml})\end{array}$ & $1.774 \pm 0.129$ & $6.211 \pm 0.197$ \\
\hline$P$ value & $<0.05$ & $<0.05$ \\
\hline $\begin{array}{l}\text { Lv-KRT17P3+mimic-NC/ IC50 } \\
(\mu \mathrm{g} / \mathrm{ml})\end{array}$ & $6.106 \pm 0.09$ & $14.16 \pm 0.138$ \\
\hline $\begin{array}{l}\mathrm{Lv}-\mathrm{KRT17P3}+\text { miR497-5p mimic/ } \\
\text { IC50 }(\mu \mathrm{g} / \mathrm{ml})\end{array}$ & $4.013 \pm 0.144$ & $12.09 \pm 0.167$ \\
\hline$P$ value & $<0.05$ & $<0.05$ \\
\hline
\end{tabular}

(D)

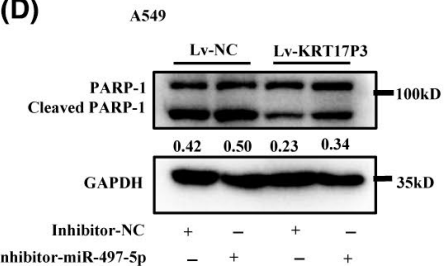

SK-MES-1

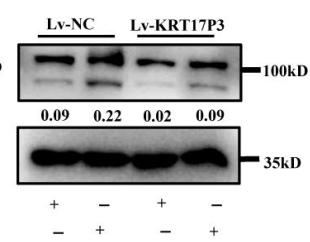

(C)
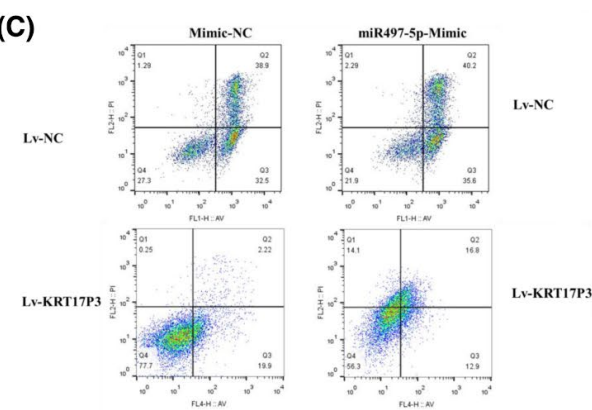
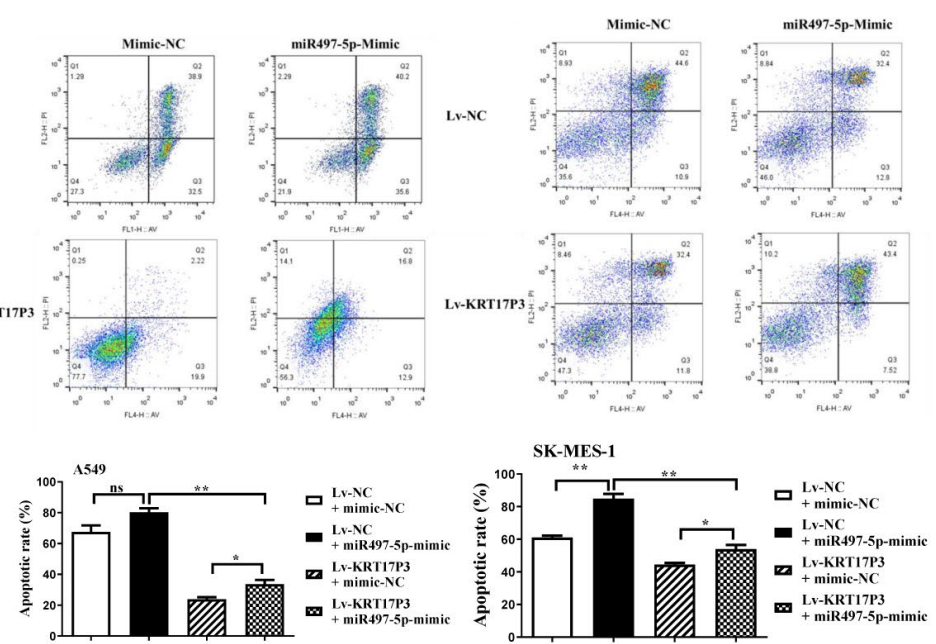

FIGURE 7 The effect of keratin 17 pseudogene 3 (KRT17P3) on cisplatin resistance is reversed by miRNA-497-5p expression. A and B, KRT17P3-overexpressing A549 and SK-MES-1 cells were transfected with miR-497-5p mimic or control mimic and exposed to various concentrations of cisplatin for $72 \mathrm{~h}$ to analyze cell viability and IC50 with CCK8 assay. C and D, Apoptosis levels were evaluated by flow cytometry (C) and Western blotting (D) 

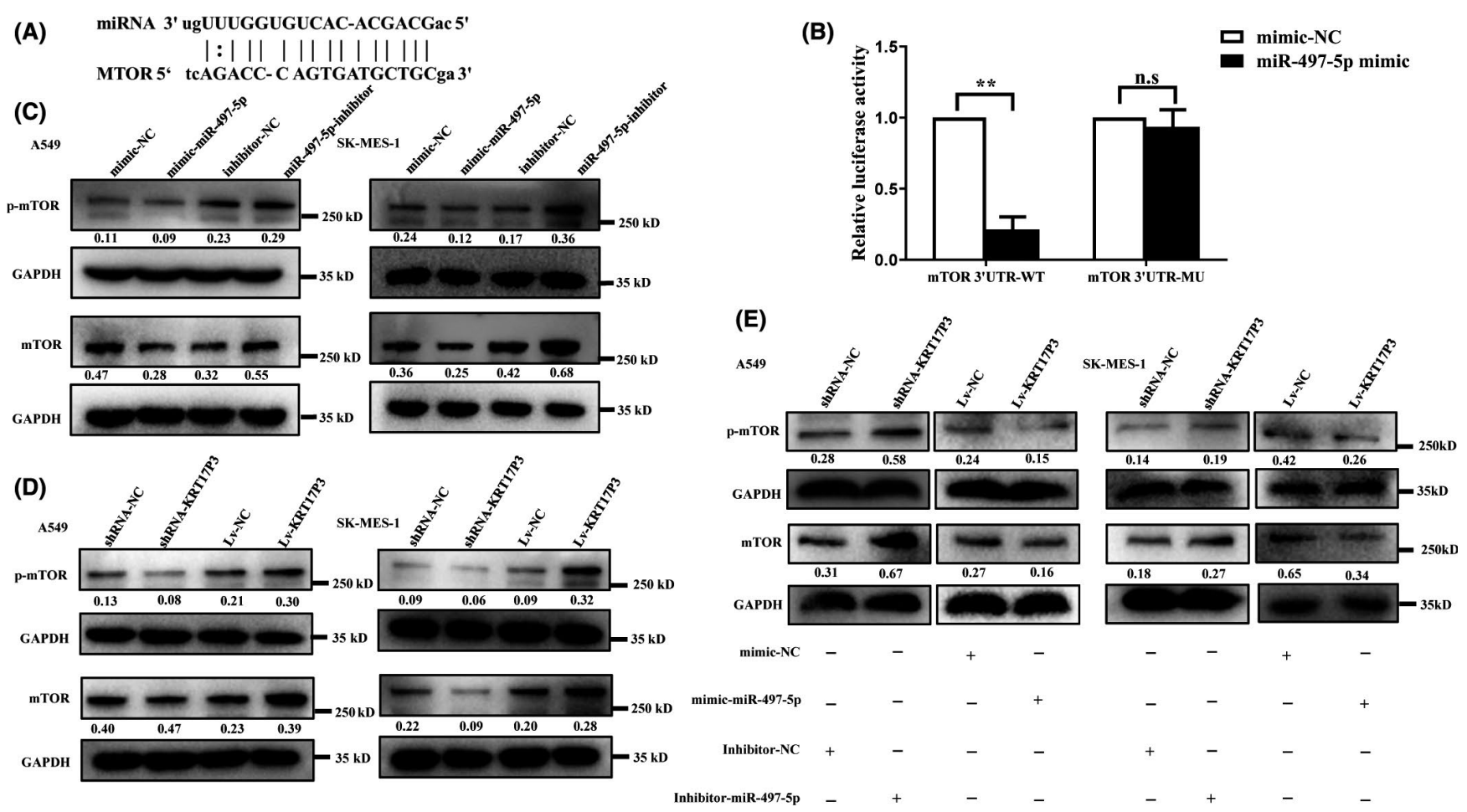

FIGURE 8 The effect of keratin 17 pseudogene 3 (KRT17P3) on cisplatin resistance is mediated in part through regulation of the miRNA-497-5p/mTOR axis. A, Sequence of miR-497-5p predicted to target mTOR. B, Dual-luciferase assay of 293T cells cotransfected with miR-497-5p mimic or mimic-NC and reporter plasmid containing wild-type or mutant mTOR 3'-UTR. C, The protein levels of mTOR and phosphorylated-mTOR in A549 and SK-MES-1 cells transfected with miR-497-5p mimic or miR-497-5p inhibitor were determined by Western blotting. D, The protein levels of mTOR and phosphorylated-mTOR in KRT17P3-overexpressing or KRT17P3-knockdown A549 and SK-MES-1 cells were measured by Western blotting. E, KRT17P3-knockdown A549 and SK-MES-1 cells or KRT17P3-overexpressing A549 and SK-MES-1 cells were transfected with miR-497-5p inhibitor or miR-497-5p mimic and then subjected to Western blotting to measure the protein levels of mTOR and phosphorylated-mTOR

\section{5 | KRT17P3 contributes to cisplatin resistance in part through regulation of the miRNA-497-5p/ mTOR axis}

Based on the above results, we sought to explore potential targets of miR-497-5p by MIRANDA (http://www.microrna.org) and TARGETSCAN (http://www.targetscan.org) algorithms. Among them, mTOR was predicted to be targeted by miR-497-5p through a recognition sequence in its $3^{\prime}$ UTR (Figure $8 \mathrm{~A}$ ). To verify this prediction, we performed luciferase reporter assays with plasmid containing the predicted wild type or mutant miR-497-5p binding site. The miR-497-5p mimic significantly weakened the luciferase activity of the mTOR 3'UTR-WT, but not in its mutant plasmid (Figure 8B). Furthermore, modulation of miR-497-5p expression changed the protein level of mTOR and phosphorylated-mTOR, as detected by Western blotting (Figure $8 \mathrm{C}$ ). These data suggest that mTOR is a direct target of miRNA-497-5p.

Next, to test whether KRT17P3 expression regulates the miRNA-497-5p/mTOR axis, we performed Western blotting assays in KRT17P3 overexpression and knockdown cells. The results verify that KRT17P3 induces the expression of mTOR and phosphorylated-mTOR, whereas KRT17P3 shRNA represses mTOR and phosphorylated-mTOR expression (Figure 8D). Furthermore, miRNA-497-5p inhibitor rescued the expression of $\mathrm{mTOR}$ and phosphorylated-mTOR in KRT17P3 knockdown NSCLC cells, while miRNA-497-5p mimic decreased the mTOR and phosphorylated-mTOR expression in KRT17P3-overexpressing cells (Figure 8E). Collectively, these data suggest that KRT17P3 enhances cisplatin resistance of NSCLC cells by regulating the miRNA-497-5p/mTOR axis.

\section{4 | DISCUSSION}

Noncoding RNAs, including IncRNAs, circRNAs, and pseudogenes, have been widely verified to participate in tumorigenesis, tumor progression, and chemoresistance. ${ }^{14}$ Several lines of evidence have demonstrated roles for pseudogenes, providing new insights into cisplatin chemoresistance. ${ }^{4,9,10}$ In a previous study, we determined that the pseudogene KRT17P3 is highly expressed in cisplatin-resistant lung cancer from 60 LUSC tissue samples. ${ }^{10}$ Recent studies have demonstrated that IncRNAs, including pseudogenes, were present in the blood of cancer patients and showed great potential as powerful and noninvasive tumor markers. ${ }^{15-17}$ In the present study, the expression of KRT17P3 in peripheral blood was significantly higher in the chemoresistant patient group compared with the chemosensitive patient group, which is consistent with the results for 60 LUSC tissue 
samples in our previous study. ${ }^{10}$ The consistent result further enhanced the reliability in the plasma examination. Exosome released by tumor cells may be one of the sources of plasma KRT17P3, and this needs to be investigated in the future. Our results indicate KRT17P3 maybe a potential noninvasive tumor marker for discriminating cisplatin-resistant and cisplatin-sensitive patients. However, the quantity of samples used in this study is still not enough. Therefore, future research will expand the sample sizes to verify its value.

To reveal the mechanism of KRT17P3 on drug resistance in NSCLC cells, initially, we explored the role of KRT17P3 in cisplatin resistance of NSCLC in vitro and in vivo. In vitro results demonstrate that KRT17P3 overexpression significantly reduces cell apoptosis and enhances cell viability, whereas KRT17P3 knockdown increases cell apoptosis and decreases cell viability upon cisplatin treatment. In a xenografted mouse model, we observed that cisplatin, in combination with sh-KRT17P3, inhibits tumor growth, thus verifying that KRT17P3 promotes the resistance of NSCLC cells to cisplatin both in vitro and in vivo.

Recent studies suggest that pseudogenes may exert functions by targeting miRNAs. ${ }^{18}$ To determine how KRT17P3 mediates cisplatin resistance in NSCLC cells, we used online algorithms to search for miRNAs with complementary base pairing to KRT17P3 and identified nine miRNA families. Among them, we focused on miR-497-5p because it is downregulated in multiple tumors and is involved in malignancy-associated processes, including cancer initiation, progression, metastasis, and chemosensitivity. ${ }^{12,19-22}$ In this study, we found that miR-497-5p inhibition was sufficient to increase cisplatin sensitivity in NSCLC cells, while miR-497-5p mimic decreased cisplatin sensitivity. Moreover, miR-497-5p mimic reversed the cisplatin resistance induced by KRT17P3 overexpression. Conversely, miR-497-5p inhibitor attenuated the cisplatin sensitivity induced by KRT17P3 knockdown. These findings suggest that KRT17P3dependent cisplatin resistance is partly mediated via miR-497-5p suppression.

We also explored the underlying mechanism by which KRT17P3 negatively regulates miR-497-5p. Luciferase reporter assay and RIP assays validated the direct binding ability of the miR-497-5p binding site to KRT17P3, as predicted by bioinformatics analysis. These results suggest that KRT17P3 and miR-497-5p directly interact, and that KRT17P3 promotes cisplatin resistance through "sponging" miR-497-5p. These data also imply that miR-497-5p may act as a potential target for therapy for patients who are resistant to cisplatin.

Notably, miR-497-5p has been shown to regulate cisplatin chemosensitivity in many types of cancer through multiple targets. ${ }^{12,22}$ In cervical cancer, miR-497-5p improves cisplatin chemosensitivity by regulating the expression of transketolase ${ }^{22}$, while in ovarian cancer, miR-497-5p increases cisplatin sensitivity by targeting the mTOR/P70S6K signaling pathway. ${ }^{12}$ In the current study, we demonstrate that mTOR is a downstream target of miR-497-5p. Treatment with miR-497-5p mimic markedly reduced mTOR expression and improved cisplatin chemosensitivity. Furthermore, luciferase assays confirmed bioinformatics predictions that the mTOR
3'-UTR is a putative target of miR-497-5p. In addition, Western blotting assays demonstrated that miR-497-5p expression suppresses protein levels of mTOR. Finally, KRT17P3 was found to modulate the expression of mTOR, which was reversed by miRNA-497-5p mimic in NSCLC cells.

mTOR has been reported as a serine-threonine kinase that is frequently dysregulated in cancer. Overexpression of mTOR has been found in various types of cancer and correlates with tumor progression and poor prognosis. Accumulating evidence demonstrates the mTOR signaling pathway is a critical regulator of drug resistance, and a multitude of mTOR inhibitors are in development to overcome it. $^{13,23-25}$ The mTOR inhibitor rapamycin can modulate P-gp1-mediated or MDR1-mediated drug resistance. ${ }^{26,27}$ Furthermore, the mTOR inhibitor $\mathrm{CCl}-779$ is able to restore cisplatin sensitivity in small-cell lung cancer cell lines that are selected for cisplatin resistance, as well as cell lines derived from patients who failed cisplatin. ${ }^{23}$ Our present study indicates that KRT17P3 repression is a potential method for restoring cisplatin sensitivity by inhibiting $\mathrm{mTOR}$, though it is likely that other mechanisms also could contribute to KRT17P3mediated chemoresistance. Moreover, the mechanism of KRT17P3 in mediating cisplatin chemoresistance in other types of cancer remains to be explored.

In conclusion, we show for the first time that KRT17P3 is involved in cisplatin resistance in NSCLC cells. Furthermore, miR-497-5p mediates cisplatin resistance exerted by KRT17P3, which involves the modulation of mTOR. Understanding the key role of "pseudogenemiRNA" modules in NSCLC may lead to the identification of new therapeutic targets for reversing cisplatin resistance and therefore warrants further investigation.

\section{ACKNOWLEDGEMENTS}

This work was supported by grants from the National Natural Science Foundation of China (grant Nos. 81572937, 81772500, and 81770082), Invigorating Health Care through Science Technology and Education (Jiangsu Provincial Medical Youth Talent. No. QNRC2016126), the Program of Nanjing Science and Technology of Nanjing Science and Technology Commission (No. 201715059).

\section{CONFLICT OF INTEREST}

There are no conflicts of interest to declare.

\section{ORCID}

Xiaoqin Yuan (iD https://orcid.org/0000-0002-3084-5624

Yong Song (iD https://orcid.org/0000-0003-4979-4131

\section{REFERENCES}

1. Bray F, Ferlay J, Soerjomataram I, Siegel RL, Torre LA, Jemal A. Global cancer statistics 2018: GLOBOCAN estimates of incidence and mortality worldwide for 36 cancers in 185 countries. CA Cancer J Clin. 2018;68:394-424.

2. Barron-Barron F, Guzman-De Alba E, Alatorre-Alexander J, et al. National Clinical Practice Guidelines for the management of nonsmall cell lung cancer in early, locally advanced and metastatic stages. Extended version. Salud Publica Mex. 2019;61:359-414. 
3. Stewart GL, Enfield KSS, Sage AP, et al. Aberrant expression of pseudogene-derived IncRNAs as an alternative mechanism of cancer gene regulation in lung adenocarcinoma. Front Genet. 2019;10:138.

4. Shih $\mathrm{J}-\mathrm{H}$, Chen $\mathrm{H}-\mathrm{Y}$, Lin S-C, et al. Integrative analyses of noncoding RNAs reveal the potential mechanisms augmenting tumor malignancy in lung adenocarcinoma. Nucleic Acids Res. 2020;48:1175-1191.

5. Yu G, Yao W, Gumireddy K, et al. Pseudogene PTENP1 functions as a competing endogenous RNA to suppress clear-cell renal cell carcinoma progression. Mol Cancer Ther. 2014;13:3086-3097.

6. Ma X, Wang B, Wang X, Luo Y, Fan W. NANOGP8 is the key regulator of stemness, EMT, Wnt pathway, chemoresistance, and other malignant phenotypes in gastric cancer cells. PLoS One. 2018;13:e0192436.

7. Zheng J, Zhang H, Ma R, Liu H, Gao P. Long non-coding RNA KRT19P3 suppresses proliferation and metastasis through COPS7A-mediated NF-kappaB pathway in gastric cancer. Oncogene. 2019;38:7073-7088.

8. Oliveira-Mateos C, Sánchez-Castillo A, Soler M, et al. The transcribed pseudogene RPSAP52 enhances the oncofetal HMGA2IGF2BP2-RAS axis through LIN28B-dependent and independent let-7 inhibition. Nat Commun. 2019;10:3979.

9. Li L, Yin JY, He FZ, et al. Long noncoding RNA SFTA1P promoted apoptosis and increased cisplatin chemosensitivity via regulating the hnRNP-U-GADD45A axis in lung squamous cell carcinoma. Oncotarget. 2017;8:97476-97489.

10. Hou Z, Xu C, Xie H, et al. Long noncoding RNAs expression patterns associated with chemo response to cisplatin based chemotherapy in lung squamous cell carcinoma patients. PLoS One. 2014;9:e108133.

11. Salmena L, Poliseno L, Tay Y, Kats L, Pandolfi PP. A ceRNA hypothesis: the Rosetta Stone of a hidden RNA language? Cell. 2011;146:353-358.

12. Xu S, Fu G-B, Tao Z, et al. MiR-497 decreases cisplatin resistance in ovarian cancer cells by targeting mTOR/P70S6K1. Oncotarget. 2015;6:26457-26471.

13. Deng J, Bai X, Feng $X$, et al. Inhibition of PI3K/Akt/mTOR signaling pathway alleviates ovarian cancer chemoresistance through reversing epithelial-mesenchymal transition and decreasing cancer stem cell marker expression. BMC Cancer. 2019;19:618.

14. Huang H, Chen J, Ding CM, Jin X, Jia ZM, Peng J. LncRNA NR2F1-AS1 regulates hepatocellular carcinoma oxaliplatin resistance by targeting ABCC1 via miR-363. J Cell Mol Med. 2018;22:3238-3245.

15. Lui KY, Peng H-R, Lin J-R, et al. Pseudogene integrator complex subunit 6 pseudogene 1 (INTS6P1) as a novel plasma-based biomarker for hepatocellular carcinoma screening. Tumour Biol. 2016;37:1253-1260.

16. Jiang $\mathrm{N}$, Meng X, Mi H, et al. Circulating IncRNA XLOC_009167 serves as a diagnostic biomarker to predict lung cancer. Clin Chim Acta. 2018;486:26-33
17. Xie Y, Zhang YI, Du L, et al. Circulating long noncoding RNA act as potential novel biomarkers for diagnosis and prognosis of non-small cell lung cancer. Mol Oncol. 2018;12:648-658.

18. Wang L, Guo Z-Y, Zhang R, et al. Pseudogene OCT4-pg4 functions as a natural micro RNA sponge to regulate OCT4 expression by competing for miR-145 in hepatocellular carcinoma. Carcinogenesis. 2013;34:1773-1781.

19. Xu GS, Li ZW, Huang ZP, et al. MiR-497-5p inhibits cell proliferation and metastasis in hepatocellular carcinoma by targeting insulin-like growth factor 1. Mol Genet Genomic Med. 2019;7(10):e860.

20. Li G, Wang K, Wang J, Qin S, Sun X, Ren H. miR-497-5p inhibits tumor cell growth and invasion by targeting SOX5 in non-small-cell lung cancer. J Cell Biochem. 2019;120:10587-10595.

21. Wang L, Jiang C-F, Li D-M, et al. MicroRNA-497 inhibits tumor growth and increases chemosensitivity to 5-fluorouracil treatment by targeting KSR1. Oncotarget. 2016;7:2660-2671.

22. Yang $\mathrm{H}, \mathrm{Wu} \mathrm{XL}, \mathrm{Wu} \mathrm{KH}$, et al. MicroRNA-497 regulates cisplatin chemosensitivity of cervical cancer by targeting transketolase. American journal of cancer research. 2016;6:2690-2699.

23. Wu C, Wangpaichitr M, Feun L, et al. Overcoming cisplatin resistance by mTOR inhibitor in lung cancer. Mol Cancer. 2005;4:25.

24. Wangpaichitr M, Wu C, You M, et al. Inhibition of mTOR restores cisplatin sensitivity through down-regulation of growth and anti-apoptotic proteins. Eur J Pharmacol. 2008;591:124-127.

25. Xu W, Wei Y, Li Y, et al. TAZ inhibition restores sensitivity of cisplatin via AKT/mTOR signaling in lung adenocarcinoma. Oncol Rep. 2017;38:1815-1821

26. Arceci RJ, Stieglitz K, Bierer BE. Immunosuppressants FK506 and rapamycin function as reversal agents of the multidrug resistance phenotype. Blood. 1992;80:1528-1536.

27. Hoof T, Demmer A, Christians U, Tummler B. Reversal of multidrug resistance in Chinese hamster ovary cells by the immunosuppressive agent rapamycin. Eur J Pharmacol. 1993;246:53-58.

\section{SUPPORTING INFORMATION}

Additional supporting information may be found online in the Supporting Information section.

How to cite this article: Hou Z, Wang Y, Xia N, Lv T-F, Yuan X, Song Y. Pseudogene KRT17P3 drives cisplatin resistance of human NSCLC cells by modulating miR-497-5p/mTOR. Cancer Sci. 2021;112:275-286. https://doi.org/10.1111/cas.14733 